\title{
Analysis of Traffic Accidents of Young Drivers in Urban Areas and Measures to Increase Safety
}

\author{
Brlek Predraga ${ }^{a}$ Krpan Ljudevita, Cvitković Ivan ${ }^{a}$, Lukačić Krunoslava \\ a University North, Department for logistics and sustainable mobility, Koprivnica, Croatia
}

\begin{tabular}{l} 
A R T I C L E I N F O \\
\hline DOI: 10.31075/PIS.66.01.05 \\
Professional paper \\
Received:25.12.2019. \\
Accepted: 17.02.2020. \\
Corresponding author: \\
predrag.brlek@unin..hr \\
\hline Keywords: \\
Traffic accident \\
Young drivers \\
Traffic safety
\end{tabular}

\begin{abstract}
A B S T R A C T
Traffic accidents are considered the first cause of death of young people, that is, young drivers. Therefore, this problem needs to be given a lot of attention. Traffic accident which include young drivers must be analyzed as an element of the system, and it is necessary to analyze and investigate potential solutions and improve all elements in order to improve the system itself. In the Republic of Croatia, for the first time in 2004, the term "YOUNG DRIVER" as a driver of a motor vehicle up to the age of 24 , who holds a driver's license issued in the Republic of Croatia, was introduced into the Road Traffic Safety Act. The share of young drivers in the population in the Republic of Croatia is $4.3 \%$. Young drivers make up about eight percent of road users, causing a total of $9.6 \%$ of traffic accidents, or $12.5 \%$ of those with fatalities. This paper will deal with traffic safety in other countries, especially in the surrounding countries. According to statistics related to road accidents, young drivers are over-represented and are at greater risk to themselves, passengers and other participants than other drivers. There is a need to respond in a timely manner and to adopt optimal measures to improve the safety of young drivers in road traffic and thus for the safety of other road users. The aim of this paper is to outline measures that would reduce the number of road accidents involving young drivers, especially in urban areas.
\end{abstract}

\section{INTRODUCTION}

Traffic accidents are considered to be the first cause of death for young people, young drivers, so this issue needs to be given close attention. Considering the characteristics of traffic safety as a system, it is necessary to analyze and improve all the elements in order to improve the system itself. Traffic accidents of young drivers also need to be analyzed as an element of the system and to explore potential solutions. The aim of the research is to analyze the state of traffic safety and to propose possible measures for improving the safety of young drivers. Given that traffic safety is applicable throughout Europe, this paper will deal with traffic safety in other countries, and especially in the surrounding countries. According to statistics related to road accidents, young drivers are over-represented and are at greater risk to themselves, passengers and other participants than other drivers.
It is questionable how young drivers are, after passing the driving test, truly ready to participate safely in the traffic system and deal with dangerous traffic situations. It is necessary to react in a timely manner and to take the necessary measures to improve the safety situation of young drivers in road traffic. Therefore, the main topic of the paper is to highlight the problem of young drivers on the roads, and the aim of this paper is to present measures that would reduce the number of traffic accidents involving young drivers.

The term "young driver" is defined differently in the surrounding countries law. Thus, for the first time in the Republic of Croatia, it is mentioned in the Road Traffic Safety Act 2004 and today it designates a driver of a motor vehicle until the age of 24 , who holds a driver's license issued in the Republic of Croatia. In the Republic of Slovenia, the term refers to the driver of a motor vehicle until the age of 21 , for the first two years after obtaining a driver's license, regardless of age at the time 
of passing the driving test. According to the national legislation, each country has its own guidelines, but it is common that all the countries observed have introduced a permissible alcohol limit of $0.0 \mathrm{~g} / \mathrm{kg}$. The only exception is Montenegro, where a limit of $0.3 \mathrm{~g} / \mathrm{kg}$ is allowed for drivers of all ages and levels of driving experience

\section{ANALYSIS OF THE SITUATION IN THE EUROPEAN UNION}

In 2017, the European Road Safety Observatory (hereinafter referred to as "ERSO") published a Traffic Safety Basic Facts report summarizing European Union-wide traffic safety statistics from 2006 to 2015, with a special issue dedicated to the demographic group of young people, ie individuals aged 18-24.

In the observed period, 52852 young people were killed in road accidents, representing $16 \%$ of all traffic related deaths in the European Union.

Table 1. Number of young people killed in traffic from 2011 to 2017, by country [1]

\begin{tabular}{|l|l|l|l|l|l|l|l|}
\hline & 2011 & 2012 & 2013 & 2014 & 2015 & 2016 & 2017 \\
\hline BG & - & - & - & - & - & - & - \\
\hline CZ & 129 & 96 & 96 & 100 & 103 & 65 & 68 \\
\hline DE & 737 & 611 & 493 & 496 & 473 & 435 & 394 \\
\hline EL & 163 & 143 & 112 & 114 & 112 & 101 & - \\
\hline HR & 81 & 48 & 58 & 36 & 45 & 42 & 48 \\
\hline IT & 496 & 423 & 404 & 369 & 379 & 352 & 306 \\
\hline HU & 73 & 42 & 50 & 59 & 61 & 47 & 44 \\
\hline AT & 76 & 84 & 56 & 59 & 72 & 56 & 46 \\
\hline RO & 251 & 231 & 220 & 177 & 207 & 195 & 196 \\
\hline SI & 17 & 19 & 22 & 9 & 16 & & \\
\hline SK & - & - & - & - & - & - & - \\
\hline
\end{tabular}

Source: European Commission, „Traffic Safety Basic Facts 2017-Main Figures“, European Commission, Directorate General for Transport, June 2017 and European Commission CARE DATABASE

The data show that in the decade studied, a dramatic fall in the number of deaths occurred in all EU countries, almost halved, which is particularly evident from the example of Estonia, which reduced the death toll by $86 \%$ in the observed period. The most common cause of accidents is speeding, or speed unsuitable for road conditions. The second most common cause of accidents is driving in the opposite or prohibited direction. Not taking any action is a third cause of accidents, which means that the driver did not react at all for any reason to avoid a collision.

Regarding the vehicle in which the car accident occurred, most of them occurred in the passenger car (65\% of all accidents). According to the classification of the roads on which the accident occurred, only $7 \%$ of accidents occurred on motorways and expressways, $92 \%$ of accidents occurred off the highways and expressways (of which $62 \%$ accidents occurred on rural roads and $30 \%$ on urban roads). while we do not have data for $1 \%$ of road accidents.

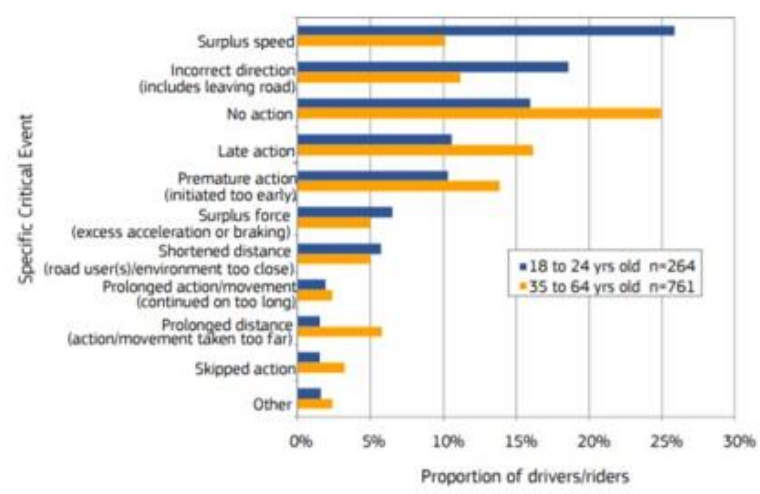

Figure 1. Overview of causes of traffic accidents, young drivers (1824 years) and other populations (35-64 years)

Source: European Commission, „Traffic Safety Basic Facts 2017-Main Figures“, European Commission, Directorate General for Transport, June 2017 and European Commission CARE DATABASE

Table 2: Traffic accidents in the period from 2006 to 2015 by type of road

\begin{tabular}{|c|c|c|c|c|c|}
\hline & \multirow[t]{2}{*}{ MOTORWAY } & \multicolumn{2}{|c|}{ NON-MOTORWAY } & \multirow[t]{2}{*}{ UNKNOWN } & \multirow[t]{2}{*}{ TOTAL } \\
\hline & & RURAL & URBAN & & \\
\hline$B G$ & $4 \%$ & $66 \%$ & $29 \%$ & & 157 \\
\hline CZ & $2 \%$ & $86 \%$ & $12 \%$ & & 103 \\
\hline $\mathrm{DE}$ & $11 \%$ & $72 \%$ & $17 \%$ & & 473 \\
\hline EL & $6 \%$ & $38 \%$ & $55 \%$ & & 112 \\
\hline HR & $4 \%$ & $36 \%$ & $60 \%$ & & 45 \\
\hline IT & $7 \%$ & $53 \%$ & $40 \%$ & & 379 \\
\hline HU & $10 \%$ & $54 \%$ & $36 \%$ & & 61 \\
\hline AT & $8 \%$ & $72 \%$ & $19 \%$ & & 72 \\
\hline RO & $2 \%$ & $38 \%$ & $60 \%$ & & 207 \\
\hline SI & $13 \%$ & $56 \%$ & $31 \%$ & & 16 \\
\hline SK & $5 \%$ & $59 \%$ & $36 \%$ & & 59 \\
\hline EU & $7 \%$ & $62 \%$ & $30 \%$ & $1 \%$ & 3.636 \\
\hline
\end{tabular}

Source: European Commission, „Traffic Safety Basic Facts 2017-Main Figures European Commission, Directorate General for Transport, June 2017 and European Commission CARE DATABASE

\section{ANALYSIS OF THE SITUATION IN THE SURROUNDING COUNTRIES, WITH REVIEW OF THE REPUBLIC OF CROATIA AND THE REPUBLIC OF SLOVENIA}

Researching statistics on road accidents for young drivers in the surrounding countries by examining publicly available documents, it is noticeable that not all countries conduct the same level of statistical analysis. e.g. Slovenia conducts the most detailed analysis with the largest number of parameters. [2]

In 2005, when the term was introduced, young By gender, $82 \%$ of all deaths are young men, which is explained by the fact that men drive motor vehicles more often, choose to travel longer distances more often, and present more aggressive driving. When analyzing the time of a car accident, they most commonly occur between $8 \mathrm{pm}$ and midnight, from $4 \mathrm{am}$ to $8 \mathrm{am}$ and between $4 \mathrm{pm}$ and $8 \mathrm{pm}$. In the observed decade, road accidents most often occurred on weekends, when the highest number of road accidents occurred between $7 \mathrm{pm}$ and $5 \mathrm{am}$ on Fridays and from midnight to 6am in the night from Saturday to Sunday. 
When analyzing the months of 2015 in which the highest number of accidents occurred, the period July and August (21\%) was highlighted, while the lowest number of accidents occurred in January and February $(13 \%)$, however, these data differ by country.

Table 3. Distribution of traffic accidents by country and month, 2015 year

\begin{tabular}{|c|c|c|c|c|c|c|c|}
\hline & Jan/Fab & Mar/Apr & May/June & Jul/Aug & Sep/Oct & Nov/Dec & Total \\
\hline BG & $8 \%$ & $16 \%$ & $15 \%$ & $28 \%$ & $18 \%$ & $15 \%$ & 157 \\
\hline CZ & $15 \%$ & $23 \%$ & $13 \%$ & $19 \%$ & $17 \%$ & $14 \%$ & 103 \\
\hline DE & $13 \%$ & $15 \%$ & $18 \%$ & $19 \%$ & $19 \%$ & $15 \%$ & 473 \\
\hline EL & $16 \%$ & $16 \%$ & $14 \%$ & $21 \%$ & $23 \%$ & $10 \%$ & 112 \\
\hline HR & $13 \%$ & $9 \%$ & $20 \%$ & $27 \%$ & $20 \%$ & $11 \%$ & 45 \\
\hline IT & $12 \%$ & $13 \%$ & $16 \%$ & $23 \%$ & $18 \%$ & $18 \%$ & 379 \\
\hline HU & $13 \%$ & $11 \%$ & $28 \%$ & $20 \%$ & $13 \%$ & $15 \%$ & 61 \\
\hline AT & $7 \%$ & $13 \%$ & $11 \%$ & $38 \%$ & $18 \%$ & $14 \%$ & 72 \\
\hline RO & $13 \%$ & $16 \%$ & $11 \%$ & $18 \%$ & $16 \%$ & $25 \%$ & 207 \\
\hline SI & $0 \%$ & $13 \%$ & $6 \%$ & $19 \%$ & $38 \%$ & $25 \%$ & 16 \\
\hline SK & $14 \%$ & $12 \%$ & $14 \%$ & $20 \%$ & $22 \%$ & $19 \%$ & 59 \\
\hline EU & $13 \%$ & $15 \%$ & $16 \%$ & $21 \%$ & $18 \%$ & $16 \%$ & 3.636 \\
\hline
\end{tabular}

Source: European Commission, „Traffic Safety Basic Facts 2017-Main Figures“, European Commission, Directorate General for Transport, June 2017 and European Commission CARE DATABASE

Drivers were involved in 11954 car accidents. In 2018, young drivers in Croatia participated in 3454 road accidents, resulting in 33 deaths and 1878 injuries. [3] Compared to 2017, positive developments were observed, i.e. a decrease in the number of accidents by 81 accidents, a decrease in the number of injured persons by 56 , but unfortunately, an increase of 3 killed more than in 2017.

In Slovenia, during 2018, young drivers participated in a total of 3721 accidents, of which 2294 were hidden. 744 accidents resulted in minor injuries, 79 more serious and 15 with fatalities. Alcohol consumption (702 accidents) are the most common causes of accidents.

According to the data of the Slovenian Traffic Safety Agency, young drivers are generally involved in the largest number of car accidents during the weekend, from $11 \mathrm{pm}$ to $6 \mathrm{am}$, while the most common causes of accidents are alcohol-fueled driving and noncompliance with traffic rules. [4]

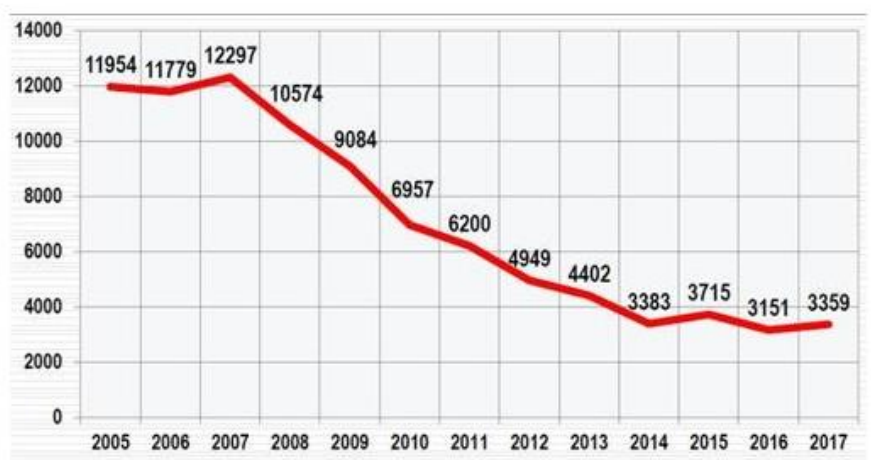

Figure 2. Number of traffic accidents caused by young drivers Source: Croatian automobile club

\section{MEASURES FOR REDUCING NUMBER OF ROAD ACCIDENTS WITH YOUNG DRIVERS}

Road user behavior is determined by a combination of factors, including the road infrastructure, road traffic regulations, levels of traffic law enforcement, in-vehicleenforcement, in-vehicle safety technology, behavior of other road traffic participants and traffic education. As this paper deals with young drivers, only behavior of traffic participants and traffic education is analyses further. Young drivers represent a greater risk everywhere and therefore prevention needs to be better addressed. It is crucial to understand young driver's mind and motives.

Research shows that attitudes about safety are formed at an early age, long before legal driving, and therefore it would also be important to target children and young adolescents. It is necessary to start educating those groups as early as possible, adjusted to their cognitive development. Early campaigns need to be introduced at even kindergarten level as a first step of shaping responsible traffic participant. Those campaigns need to be followed by more extensive ones as target group's age elevate. Interdisciplinary approach needs to be used while creating campaigns and should include various professionals like traffic experts and children psychologist to create campaigns that will result with maximum effect.

Besides that, some experts advocate the principle of "sustainable safety" [6] on roads is based on the thesis that the entire transport and transport system is based on human constraints. The road infrastructure prevents the use of roads that involve large differences in direction, speed and mass and directs the user towards safe behavior.

The vehicles are built to simplify the task of driving and offer crash protection. Road users are educated and informed and their behavior is regularly tested. "Sustainable safety" is based on the five principles, and two of these five principles are based on the "selfexplanatory road" and "forgiving road environment". [7]

\section{CONCLUSION}

Traffic safety is a combination of human being, vehicle and road. These factors are interdependent and in order to maximize traffic safety, attention must be paid to each of these factors. This paper focused on the human factor, more specifically on the category of young drivers. Obtaining a driver's license is wrongly perceived as the last step in training to operate a motor vehicle independently. Statistics say that young drivers are a particularly vulnerable category. In order to educate a safe, defensive and attentive driver, it is important to begin to instill a knowledge of traffic culture from an early age. 
Thus, a candidate for a driver or a young driver will have increased competencies when faced with a driving test and self-driving situation. After a brief cross-section of statistics for the European Union and in the surrounding countries, and the proposal of measures to increase the safety of young drivers, this paper reiterates the need to introduce a transport culture from an early age, but also to understand that the education of young drivers is a process that different professions need to approach in synergy, interdisciplinary. Only by acting together will the educational process result in the creation of a responsible driver, which will also reduce the frequency of traffic accidents and indirectly the number of casualties and fatalities in traffic.

\section{References}

[1] European Commission, „Traffic Safety Basic Facts 2017-Main Figures" , European Commission, Directorate General for Transport, June 2017 and European Commission CARE DATABASE

[2] http:// http://nesrece.avp-rs.sil, approached September 2019

3] Ministry of Internal Affairs Croatia, Road Safety Newsletter, 2019

[4] http:// http://nesrece.avp-rs.sil, approached September 2019

[5] https://www.hak.hr/ approached September 2019

[6] Tresure, M. "Principles of sustainable safety in the Netherlands", Vision Zero 2016.

[7] Brlek, P. "Method of sanation of dangerous sports on roads with the use od geo-referenced video", Doctoral thesis, Faculty of transport and traffic sciences, Zagreb, 2017 